\title{
Perioperative Management of Pancreaticoduodenectomy: Avoiding Admission to the Intensive Care Unit
}

\author{
Walid Faraj ${ }^{a}$ Deborah Mukherji ${ }^{\mathrm{b}} \quad$ Ahmad M. Zaghal ${ }^{\mathrm{a}}$ Hussein Nassar ${ }^{\mathrm{a}}$ \\ Farah H. Mokadem ${ }^{c}$ Samar Jabbour ${ }^{c}$ Chakib M. Ayoub ${ }^{c}$ \\ Marwan S. Rizk ${ }^{c}$ Mariam Kanso $^{a}$ Rola F. Jaafar ${ }^{a}$ Nigel Heaton ${ }^{d}$ \\ Mohamad J. Khalifea \\ aLiver Transplantation and Hepatopancreaticobiliary Surgery, Department of General \\ Surgery, American University of Beirut Medical Center, Beirut, Lebanon; ${ }^{b}$ Department of \\ Oncology, American University of Beirut Medical Center, Beirut, Lebanon; ' $D$ epartment of \\ Anesthesia, American University of Beirut Medical Center, Beirut, Lebanon; ${ }^{d}$ Institute of \\ Liver Studies, Kings College Hospital, London, UK
}

\author{
Keywords \\ Pancreaticoduodenectomy · Pancreatic cancer · Outcome
}

\begin{abstract}
Introduction: With the advancement in surgical expertise at high-volume centers and advances in perioperative management, pancreaticoduodenectomy is becoming safer and remains the gold standard for treating periampullary pathologies. We describe our experience in optimizing perioperative management in order to avoid admission to the intensive care unit and improve outcomes. Method: Retrospective data were collected on 370 surgical patients who underwent a pancreaticoduodenectomy between the years 1994 and 2016. Results: Of the 370 patients, 200 operated between 2009 and 2016 did not require intensive care admission, blood transfusion, or central line insertion. The results were compared between different time intervals: before the year 1998, between the years 1998 and 2008, and between the years 2009 and 2016. The median blood loss dropped from 800 to 400 to $300 \mathrm{~mL}$, respectively. The median operative time also dropped from 360 to 335 to $215 \mathrm{~min}$, respectively. In addition, the median length of hospital stay decreased from 25 to 16 to 7 days, respectively. Conclusion: With the centralization of pancreaticoduodenectomy in high-volume centers and with specialized surgeons performing the surgery, there is a significant decrease in the onset of postoperative complications with a lesser need for blood transfusions and, subsequently, better recovery of patients without the need for intensive care unit admission.
\end{abstract}




\section{Introduction}

Pancreaticoduodenectomy is a complex surgical procedure indicated for the management of both benign and malignant diseases of the periampullary area [1,2]. It is considered a highrisk surgical procedure in terms of morbidity and mortality with lower operative complication rates in high-volume centers [3]. The complex anatomy and blood supply of the pancreaticoduodenal area contribute to the technical difficulties and prolonged operative stress induced by the surgery. The procedure is associated with major complications, including pancreatic and bile leaks, delayed gastric emptying, secondary hemorrhage from pseudoaneurysms, and sepsis [4].

Central to the complications is the incidence of leak from the pancreatic duct (PD) anastomosis. Regardless of whether the stomach or jejunum are utilized, major leak rates are estimated to be greater than or equal to $4 \%$ and are associated with a high mortality rate. The overall incidence of PD leaks is $10-20 \%$. Risk factors for PD leaks include a small PD, soft pancreas and benign or ampullary disease, prolonged surgery, and diabetes mellitus [5-7]. However, with the advancement in surgical experience, improvements in perioperative management and modern operative techniques, pancreaticoduodenectomy has become safer and considered as the gold standard for the treatment of periampullary pathologies. In highvolume centers, the mortality rate is less than $5 \%$, whereas centers that perform the procedure less frequently have a higher complication rate with the mortality rates reaching $20 \%[8,9]$. The well-documented discrepancy in mortality rates between high- and low-volume centers has been attributed to the consistency of perioperative care and early recognition and effective management of early complications such as PD leak and bleeding.

Our institution is a leading tertiary referral center in the Middle East and a center of excellence in many specialties, including oncology and interventional radiology. We present our latest 200 pancreaticoduodenectomies performed with no intensive care admission, no blood transfusion, and no central line insertion. The aim of our study is to advocate for centralization of pancreaticoduodenectomy with standardization of the perioperative care, intraoperative technique, and follow-up.

\section{Materials and Methods}

Following institutional review board approval, we performed a retrospective data analysis review of all medical charts of patients who underwent pancreaticoduodenectomies at the American University of Beirut Medical Center (AUBMC) from 1994 to 2016, inclusive. Of the 370 patients reviewed, we identified 200 patients who underwent pancreaticoduodenectomies without admission to intensive care, did not require blood transfusion, and without the insertion of a central line. Of interest, these 200 patients underwent surgery between 2009 and 2016, inclusive.

The parameters collected were patient demographic data, clinical presentation, preoperative lab results, and the presence of comorbidities with an American Society of Anesthesiology (ASA) score, past medical history, intraoperative parameters, postoperative complications, pathology results, causes of reoperations, and postoperative deaths. Our criteria for resectability of pancreatic cancer and periampullary neoplasms included the absence of distant metastases, absence of local tumor extension to the celiac axis and hepatic artery, lack of involvement of the superior mesenteric vasculature, and the feasibility of resection dictated by the preoperative diagnostic imaging.

Fifteen patients were found to have borderline resectability with no distant metastatic disease. The decision in our multidisciplinary meeting was to go for neoadjuvant chemotherapy. They all received FOLFIRINOX and only 10 patients showed a good radiological response with downstaging for resectability. Our aim was to achieve R0 resections, decrease lymph node metastasis, and ensure longer disease-free survival and lower recurrence rates. 
Table 1. Standardized surgical procedure details

a Right subcostal incision (15-17 cm) extended sometimes to the level of the xyphoid process; the liver and peritoneum are examined for metastasis, in the event of identifying suspicious lesions; an intraoperative frozen section is taken; if the biopsy is positive for cancer, the operation is aborted and a single or double bypass is performed

b The pancreaticoduodenectomy starts with a retrograde cholecystectomy and dissection of the porta hepatis, ligation and division of the common bile duct; the tissues around the portal vein are carefully separated, divided, and ligated with care taken that a possible replaced right hepatic artery off the superior mesenteric artery is present

c Dissection of the hepatic artery starts by dissecting the lymph nodes anterior to it; once identified, the right gastric and gastroduodenal arteries are divided and suture ligated

d Antrectomy (distal gastrectomy) is performed using a stapler (staple lines are reinforced using 3/0 Vicryl sutures), followed by the creation of a tunnel posterior to the pancreatic neck between the portal and superior mesenteric veins; two silk sutures are applied at the lateral edges of the resection; the pancreas is then cut using cautery with careful identification of the PD; kocherization of the duodenum is performed up to the level of the inferior vena cava; dissection and removal of retrocaval lymph nodes is performed at this stage; in all patients, energy devices are used for vessel and tissue sealing

e The duodenum is cut at the duodenojejunal junction (around 7-10 $\mathrm{cm}$ from the ligament of Treitz) using a stapler

f The specimen is finally removed after dissection and ligation of all venous and arterial tributaries of the uncinate process to the portal and superior mesenteric vein and artery

Standardized Operative Protocol

The Enhanced Recovery after Surgery (ERAS) protocol was implemented between 2009 and 2016 in an effort to enhance patients' recovery. All 200 pancreaticoduodenectomies were performed by 2 specialized hepatobiliary surgeons. The surgical technique was standardized and performed the same way in all patients as described in Table 1. In addition, no central line insertion was performed, with venous access being obtained through two large bore peripheral (14 or 16 Fr) intravenous lines and one arterial line.

\section{Results}

\section{Demographics and Clinical Presentation}

The median age was 61 years (range 29-82), with 132 (66\%) males and 68 (34\%) females. The median body mass index was 27.1 (range 15.6-43.7). Of the 200 patients, 75 were smokers (37.5\%). The median ASA score was 2 (range 1-4). The most common presenting symptom was obstructive jaundice in $75 \%$ of the patients. Other symptoms included abdominal pain, weight loss, nausea/vomiting, and ascites in $2 \%$. Forty-eight patients had a positive family history of cancer, 16 of which were pancreatic cancer.

\section{Medical Management and Radiological Modalities}

With the emergence of new data favoring the neoadjuvant treatment of borderline resectable pancreatic tumors, more patients have been operated. Of the 200 patients, 10 received neoadjuvant chemotherapy for size reduction prior to operation. Ninety-two patients (46\%) required preoperative biliary stenting; of these, 88 had preoperative endoscopic retrograde cholangiopancreatography and 4 had percutaneous biliary stent placement. Eighteen patients required preoperative blood transfusion due to severe anemia. All patients were evaluated preopera- 
Table 2. Preoperative laboratory results

\begin{tabular}{lll}
\hline & Median & Range \\
\hline Hemoglobin, g/dL & 12.5 & $7.2-18.9$ \\
Albumin, g/L & 35 & $17-51$ \\
Total bilirubin, mg/dL & 2.8 & $0.1-33.6$ \\
Aspartate aminotransferase, IU/L & 52 & $7-822$ \\
Alkaline phosphatase, IU/L & 278 & $22-2,337$ \\
International normalized ratio, IU & 1.1 & $0.75-3.2$ \\
Carbohydrate antigen 19-9, U/mL & 76 & $0.3-32,000$ \\
\hline
\end{tabular}

tively for deep vein thrombosis risk, all were put on sequential compressive devices, and patients with a high body mass index received low molecular weight heparin. Tazocin (piperacillin/tazobactam) was the standard of care for all patients for antibiotic prophylaxis.

The preoperative radiological workups included: abdominal ultrasound performed in 70 patients (23.3\%). Abdominal computed tomography scan in 176 patients (88\%), magnetic resonance imaging in 24 patients $(12 \%)$, and positron emission tomography scan in 7 patients (those were performed at outside institutions as it is not the standard of care in ours). Endoscopic ultrasonography was performed in 101 patients (50\%).

\section{Preoperative Laboratory Values}

Table 2 provides details of the preoperative laboratory values. Before 1998, pancreaticoduodenectomies were performed by general surgeons at our institution. The median length of hospital stay was 25 days (range 10-60) and the postoperative mortality was between 15 and 20\%. The median blood loss was $800 \mathrm{~mL}$ (range 500-3,000), and the median operative time was 360 min (range 310-575).

In 1998, a specialized hepatobiliary surgeon joined the department of surgery and performed the majority of pancreaticoduodenectomies. The surgical outcomes were significantly improved with a fall in the mortality rate to less than $5 \%$. The median length of hospital stay improved significantly to 16 days (range 9-38). In addition, there was a significant improvement in terms of morbidity and postoperative mortality of the patients. The median blood loss dropped to a mean of $400 \mathrm{~mL}$ (range 300-2000), and the median operative time fell to 335 min (range 180-470).

In 2009 , a second specialist hepatobiliary surgeon joined the program. All subsequent Whipple procedures were performed jointly by 2 experienced surgeons specialized in liver transplant and hepatobiliary surgery with standardized preoperative, intraoperative, and postoperative management. The mortality rate has also fallen significantly to less than $1.5 \%$. The median length of hospital stay is now 8 days (range 6-29). The median blood loss is now $300 \mathrm{~mL}$ (range 100-1,500), and the median operative time improved significantly from 360 to 210 min (range 150-360; Table 3).

The pancreatic reconstruction was performed via a pancreaticojejunal duct-to-mucosal anastomosis in 190 patients (95\%), and pancreaticojejunal invagination (all in a retro-colic fashion with a small drain [5 Fr] inserted in all duct to mucosa anastomosis) in 10 patients (5\%). The size of the pancreatic duct was greater than $6 \mathrm{~mm}$ in $50(25 \%)$ patients, between 3 and $6 \mathrm{~mm}$ in $129(64 \%)$ patients, and less than $3 \mathrm{~mm}$ in $21(10 \%)$ patients. The pancreatic gland texture was soft in $28 \%$ of patients, intermediate in $47 \%$ of patients, and hard in $25 \%$ of patients. Postoperative pancreatic fistula was defined as a leak of more than $50 \mathrm{~mL}$ of amylase-rich fluid per day through the surgical drains for 10 or more days following the operation, or pancreatic anastomotic disruption demonstrated radiographically. 
Table 3. Comparison between 3 different periods of time

\begin{tabular}{llcc}
\hline & $1994-1998$ & $1999-2008$ & $2009-2016$ \\
\hline Length of hospital stay, days & 25 & 16 & 8 \\
Postoperative mortality, \% & 25 & 7 & 1.5 \\
Median blood loss, mL & 800 & 400 & 300 \\
Median operative time, min & $360(310-575)$ & $335(180-470)$ & $210(150-360)$ \\
\hline
\end{tabular}

Table 4. Pancreatic gland texture, PD size, and types of anastomotic reconstruction

$\begin{array}{lc}\text { Pancreatic gland texture, } \% & \\ \text { Soft } & 28 \\ \text { Indurated } & 47 \\ \text { Hard } & 25 \\ \text { PD size gland texture, } n(\%) & 21(10) \\ \quad<3 \mathrm{~mm} & 129(64) \\ \quad 3-6 \mathrm{~mm} & 50(25) \\ >6 \mathrm{~mm} & \\ \text { Pancreatic anastomosis, } n(\%) & 190(95) \\ \quad \text { Duct to mucosa } & 10(5) \\ \text { Invagination } & \\ \text { Hepaticojejunostomy, } n(\%) & 164(82) \\ \text { Interrupted } & 36(18) \\ \text { Continuous } & 200(100)\end{array}$

Of the 200 pancreaticoduodenectomy performed, 41 (21\%) patients developed pancreatic fistulas, with $25(61 \%)$ grade A, $10(24 \%)$ grade B, and $6(15 \%)$ grade C, as defined in the literature [10]. All patients who developed pancreatic fistula grade $\mathrm{C}$ required percutaneous drainage and 3 patients required relaparotomy to reassess the anastomosis. The median drain fluid amylase level on postoperative day 3 was $56 \mathrm{U} / \mathrm{L}$ (range 11-39,350), and on day 5 was $27 \mathrm{U} / \mathrm{L}$ (range $0-14,295$ ). The median amount of pancreatic drain fluid on postoperative day 3 was $224 \mathrm{~mL}$ (range 10-715), and on day 5 was $200 \mathrm{~mL}$ (range 20-3,100). The range of the number of days for removal of the pancreatic drain was 2-50 days and few patients were discharged home with the pancreatic drain. The abdominal drain was removed when the drain fluid amylase concentration was below $100 \mathrm{U} / \mathrm{L}$, or the drainage was less than $50 \mathrm{~mL}$ per day.

The hepaticojejunostomy was performed in a retro-colic interrupted fashion in 164 (82\%) patients and continuous in 36 (18\%). The gastric anastomosis was performed as a gastrojejunostomy in an antecolic fashion in all patients using a stapler. All anastomosis (pancreatic, hepatic, and gastric) were performed through one jejunal limb with a distance of $15 \mathrm{~cm}$ from the pancreatic to the biliary anastomosis (we believe that it will decrease the bile leak in case of pancreatic leakage; Table 4).

\section{Discussion}

We have demonstrated that the centralization of pancreaticoduodenectomy in this highvolume center, where it is performed by specialized hepatobiliary surgeons, as well as the standardization of the perioperative care has resulted in a significant decrease in the inci- 
dence of postoperative complications. We have also observed a reduced need for blood transfusions and, subsequently, better recovery of patients without the need for central line insertion and intensive care unit admission postsurgery. Balzano et al. [11] previously showed that the operative mortality after pancreaticoduodenectomy decreases progressively with increasing hospital volume. It was demonstrated that a center is considered low volume if it conducts 5 or fewer operations annually. A center performing more than 14 cases annually was considered a high-volume center; it was also evident that the higher numbers were associated with better outcomes [12].

Specialized hepatobiliary centers with high/medium volume loads have much lower complication rates compared to low-volume centers. This was reflected with low reoperation rates $(10.3,10.7$ vs. $16.7 \%, p=0.0002)$ and reduced lengths of stay $(23.2,22.0$ vs. $31.6 \%, p<0.0001$ ) compared to low-volume centers. The mortality rates ranged from 1.8 to $4 \%$ in high-volume centers and 3.5 to $16 \%$ in low-volume centers [13-16]. Improvement in the perioperative outcomes have been associated with an increased pancreaticoduodenectomy volume at high-volume centers. Single surgeon experience is one of the important determinants of overall morbidity. However, there are comparable outcomes among experienced surgeons irrespective of annual volume. There are improved outcomes in terms of morbidity, especially the pancreatic leak rate between experienced and less experienced surgeons [16].

Søreide et al. [16] reported their experience in pancreatic surgeries from Norway, with evolution and improvement over time in the period between 1986 and 2012. Indications and postoperative outcomes, including mortality, were investigated. Results showed that the transition to university hospital and increase in volume led to significant improvements in several performance metrics, including reduced postoperative mortality with a reduction in 30 -day mortality from 16.1 to $3.5 \%(p=0.012)$. Over time, significant reductions in median hospitalization time (19 vs. 12 days, $p<0.001$ ), reoperation rate (37.1 vs. 8.4\%, $p<0.001$ ), and median ICU stay ( 3 vs. 0 days, $p<0.001$ ) were observed [17].

Seppänen et al. [17] reported a $2.1 \%$ mortality rate following pancreaticoduodenectomy between 2000 and 2013. With the centralization of pancreatic surgery in southern Finland, patients with pancreatic ductal adenocarcinoma survived markedly longer than in the early 1990s [18].

The American Cancer Society recommends that all pancreaticoduodenectomies should be performed at high-volume centers with specialized hepatobiliary teams [19]. Emphasis on multidisciplinary evaluations and clinical decision-making such as preoperative examinations, operative care, and perioperative management has improved the surgical management of patients with hepatobiliary diseases. Few studies have analyzed the impact of surgeon volume on the outcome healthcare expenditures after pancreaticoduodenectomy $[19,20]$.

Our data show that there was a significant improvement in the mortality and morbidity rates when the procedure was performed by 2 specialized hepatobiliary surgeons. This was reflected in all parameters, including operative time, blood loss, and length of hospital stay. The effect of a specialized hepatopancreatobiliary surgeon in a high-volume center resulted in an over $50 \%$ decrease in mortality and morbidity compared to a low-volume surgeon at a low-volume center. The effect of 2 surgeons operating together led to a decrease in the anesthesia time and blood loss/transfusions, and therefore potentially decreased mortality. In view of this, and with the standardization of the surgical technique, central line insertion was deferred, intensive care unit admission postsurgery was not required, and blood transfusion was rarely needed. Both of our specialist surgeons have undertaking advanced training in the UK and have maintained academic links with teams in the UK. Our department of surgery at the American University of Beirut Medical Center has links with Kings College Hospital London, with annual training electives offered to surgical trainees. 
In conclusion, high-volume surgeons with specialist training are associated with better patient outcomes. Standardization of surgical technique, perioperative anesthesia management, and ERAS protocol can safely transform a complex surgical procedure to a simplified operation with desirable outcomes.

\section{Statements of Ethics}

The requirement for consent to participate was waivered as this study involved retrospective data collection; the Institutional Review Board (IRB) approved the review of all pancreaticoduodenectomies performed between 2014 and 2016, and therefore no consent was obtained. The IRB committee that reviewed this application is the American University of Beirut IRB and the reference number is IRB00003107.

\section{Disclosure Statement}

The authors have no conflicts of interest to declare.

\section{Funding Sources}

There are no sources of funding in relation to this work.

\section{Author Contributions}

W.F. and M.J.K. proposed the study. S.J., M.S.R., F.H.M., and C.M.A. performed the research and wrote the first draft. D.M., A.Z., and H.N. drafted the work and revised the manuscript critically. M.J.K. and R.F.J. analyzed and interpreted the data. N.H. reviewed the manuscript. All authors contributed to the design and interpretation of the study and to further drafts. W.F. is the guarantor.

\section{References}

1 Romano G, Agrusa A, Galia M, Di Buono G, Chianetta D, Sorce V, et al. Whipple's pancreaticoduodenectomy: surgical technique and perioperative clinical outcomes in a single center. Int J Surg. 2015 Sep;21(suppl 1):S68-71.

2 Richter A, Niedergethmann M, Sturm JW, Lorenz D, Post S, Trede M. Long-term results of partial pancreaticoduodenectomy for ductal adenocarcinoma of the pancreatic head: 25-year experience. World J Surg. 2003 Mar;27(3):324-9.

3 Birkmeyer JD, Finlayson SR, Tosteson AN, Sharp SM, Warshaw AL, Fisher ES. Effect of hospital volume on in-hospital mortality with pancreaticoduodenectomy. Surgery. 1999 Mar;125(3):250-6.

4 Dicitore A, Caraglia M, Gaudenzi G, Manfredi G, Amato B, Mari D, et al. Type I interferon-mediated pathway interacts with peroxisome proliferator activated receptor- $\gamma$ (PPAR- $\gamma$ ): at the cross-road of pancreatic cancer cell proliferation. Biochim Biophys Acta. 2014 Jan;1845(1):42-52.

5 Larsen M, Kozarek R. Management of pancreatic ductal leaks and fistulae. J Gastroenterol Hepatol. 2014;29(7): 1360-70.

6 Ridolfi C, Angiolini MR, Gavazzi F, Spaggiari P, Tinti MC, Uccelli F, Madonini M, Montorsi M, Zerbi A. Morphohistological features of pancreatic stump are the main determinant of pancreatic fistula after pancreatoduodenectomy. BioMed Res Int. 2014;2014:641239. https://doi.org/10.1155/2014/641239.

7 Srivastava S, Sikora SS, Pandey CM, Kumar A, Saxena R, Kapoor VK. Determinants of pancreaticoenteric anastomotic leak following pancreaticoduodenectomy. ANZ J Surg. 2001 Sep;71(9):511-5.

8 Winter JM, Cameron JL, Campbell KA, Arnold MA, Chang DC, Coleman J, et al. 1423 pancreaticoduodenectomies for pancreatic cancer: A single-institution experience. J Gastrointest Surg. 2006 Nov;10(9):1199-210.

9 Sheets SS. Pancreas cancer. Surveillance, Epidemiology, and End Results Program (SEER). Bethesda: National Cancer Institute; 2014.

10 DeOliveira ML, Winter JM, Schafer M, Cunningham SC, Cameron JL, Yeo CJ, et al. Assessment of complications after pancreatic surgery: A novel grading system applied to 633 patients undergoing pancreaticoduodenectomy. Ann Surg. 2006 Dec;244(6):931-7. 


\section{Tumors}

\begin{tabular}{l|l}
\hline DOI: 10.1159/000502887 & $\begin{array}{l}\text { @ } 2019 \text { S. Karger AG, Basel } \\
\text { www.karger.com/gat }\end{array}$ \\
\hline
\end{tabular}

Faraj et al.: Perioperative Management of Pancreaticoduodenectomy

11 Balzano G, Zerbi A, Capretti G, Rocchetti S, Capitanio V, Di Carlo V. Effect of hospital volume on outcome of pancreaticoduodenectomy in Italy. Br J Surg. 2008 Mar; 95(3):357-62.

12 Cheng Q, Zhang B, Zhang Y, Jiang X, Zhang B, Yi B, et al. Predictive factors for complications after pancreaticoduodenectomy. J Surg Res. 2007 May;139(1):22-9.

13 Kakizawa N, Noda H, Watanabe F, Kamiyama H, Kato T, Ichida K, et al. Instruction of expert surgeon secures favorable outcomes after standard pancreaticoduodenectomy performed by less experienced surgeons. Hepatogastroenterology. 2014 Jan-Feb;61(129):203-7.

14 Rosemurgy AS, Bloomston M, Serafini FM, Coon B, Murr MM, Carey LC. Frequency with which surgeons undertake pancreaticoduodenectomy determines length of stay, hospital charges, and in-hospital mortality. J Gastrointest Surg. 2001 Jan-Feb;5(1):21-6.

15 Schmidt CM, Turrini O, Parikh P, House MG, Zyromski NJ, Nakeeb A, et al. Effect of hospital volume, surgeon experience, and surgeon volume on patient outcomes after pancreaticoduodenectomy: a single-institution experience. Arch Surg. 2010 Jul;145(7):634-40.

16 Søreide JA, Sandvik OM, Søreide K. Improving pancreas surgery over time: performance factors related to transition of care and patient volume. Int J Surg. 2016 Aug;32:116-22.

17 Seppänen H, Juuti A, Mustonen H, Haapamäki C, Nordling S, Carpelan-Holmström M, et al. The results of pancreatic resections and long-term survival for pancreatic ductal adenocarcinoma: a single-institution experience. Scand J Surg. 2017 Mar;106(1):54-61.

19 The American Cancer Society. Surgery for Pancreatic Cancer. March 14, 2016. Atlanta: ACS.

19 Mathur A, Luberice K, Ross S, Choung E, Rosemurgy A. Pancreaticoduodenectomy at high-volume centers: surgeon volume goes beyond the leapfrog criteria. Ann Surg. 2015 Aug;262(2):e37-9.

20 Rosemurgy A, Cowgill S, Coe B, Thomas A, Al-Saadi S, Goldin S, et al. Frequency with which surgeons undertake pancreaticoduodenectomy continues to determine length of stay, hospital charges, and in-hospital mortality. J Gastrointest Surg. 2008 Mar;12(3):442-9. 